\title{
Reasoning and Reading in Adults. A New Reasoning Task for Detecting the Visual Impendance Effect
}

\author{
Elpida Panagiotidou, Francisca Serrano, and Sergio Moreno-Rios \\ The Mind, Brain and Behavior Research Center (CIMCYC-UGR). Faculty of Psychology, University of Granada
}

\section{KEYWORDS}

visual impedance, transitive reasoning, visual deductive task, reading difficulties

\begin{abstract}
The visual impedance hypothesis states that at the time of reasoning, the reading context provokes visual images, which may add irrelevant details to an inference and thus could hamper reasoning. This study aims to create a new visual version of a reasoning task, similar to the traditional propositional task of relational syllogisms, but based on visuospatial components. Using such a task, it would be possible to investigate the deductive ability of relational inferences in tests without the need for reading. Two reasoning tasks were used and measures of working memory, visuospatial memory, intelligence, and reading comprehension were taken. The participants were 61 university students without reading difficulties. Results show that both versions of the reasoning task work similarly in finding the main reasoning effects expected. Findings support the visual impedance effect, that is, fewer correct responses in problems with imaginable contents than with neutral ones. They indicate that this new visual task could be used to explore reasoning skills without reading being involved, and this would be useful for testing reasoning in people both with and without reading difficulties.
\end{abstract}

ABSTRACT

\section{INTRODUCTION}

Reading and reasoning skills appear to be related, especially regarding reading comprehension, which involves making inferences, among other reasoning skills (Graesser, Singer, \& Trabasso, 1994).

This relationship is also supported by findings from the scientific literature about reasoning. For instance, in deductive reasoning, Osana, Lacroix, Tucker, Idan, and Jabbour (2007) tested the association between specific types of text and reasoning, showing that the type of text affects reasoning skills. Likewise, some studies have investigated differences in reasoning task performance as a function of reading skills (Bacon \& Handley, 2010; Bacon \& Handley, 2014; Bacon, Parmentier, \& Barr, 2013; Cromley, Snyder-Hogan \& Luciw-Dubas, 2010). Moreover, it is shown that practice in extracting inferences from texts improves reading comprehension skills (Cromley et al., 2010).

Following the theory of mental models (Johnson-Laird, 1983; Johnson-Laird, 2006), when people make inferences, they construct mental models that keep the structure of the situations represented by the premises. Mental models can be created from perception, imagination, or by understanding of the premises, and they can provoke visual images. Equally, they can be abstract, representing conditions that cannot be visualised (Johnson-Laird \& Byrne, 2002). When reasoning, some people may rely on irrelevant visual images instead of on abstract spatial models to carry out relational inferences (Knauff \& JohnsonLaird, 2002).

Knauff and Johnson-Laird (2002) found that "the relationships that elicit visual images containing details that are irrelevant to an inference should impede the process of reasoning" (p. 364). This obstacle to reasoning is called the visual imagery impedance hypothesis. Thus,

Corresponding author: Francisca Serrano, The Mind, Brain, and Behavior Research Center, (CIMCYC-UGR), University of Granada, Campus de Cartuja, 18017, Granada, Spain.

E-mail:fdserran@ugr.es 
relationships that are easily visualised but difficult to imagine spatially could somehow interfere with reasoning in comparison with other types of relationships, while visuospatial relationships would facilitate it (Knauff \& Johnson-Laird, 2002). Moreover, it is demonstrated that "depending on their cognitive style and how easily they are able to use imagery during reasoning, people are influenced in different ways by the imaginability of the content of reasoning problems" (Gazzo Castaneda \& Knauff, 2013, p. 2378). Specifically, the results of Gazzo Castaneda and Knauff (2013) indicate that people who prefer to envisage the premises of reasoning problems also attempt to envisage nonvisual problems, which is why they present the visual impedance effect (also agreed by Knauff, 2018). Sato, Sugimoto, and Ueda (2017) recently showed the visual impedance effect in reasoning using real objects which could also be moved, supporting the idea that irrelevant details may impede reasoning.

Some of the existing research relating reasoning and reading and the visual impedance effect has been carried out in samples of people with reading disabilities (Bacon \& Handley, 2010; Bacon et al., 2013). Some results suggest that people with dyslexia would not show the visual impedance effect (Knauff \& Johnson-Laird, 2002), as opposed to participants without such difficulties. More precisely, the results showed that people with dyslexia always used a visual strategy, by representing vividly in their written protocols the information of the premises, even when the adjectives of the premises were not imaginable. This could indicate that people with dyslexia may use visual strategies in reasoning tasks, while people without dyslexia would rely more on spatial or propositional strategies (Bacon \& Handley, 2010; Bacon \& Handley, 2014).

People with dyslexia may tend to rely on visual strategies instead of propositional ones to try and overcome their written language disabilities (MacCullagh, Bosanquet, \& Badcock, 2017). It also suggests that people with dyslexia have difficulty finding a suitable strategy, as they seem to insist on a sequential approach (Bacon et al., 2013), which does not help them in successfully solving reasoning problems.

Moreover, the results of the visual impedance effect in the study by Bacon and Handley (2010) came from propositional problems that had to be read, so that people with reading difficulties would have had trouble due to the obstacle posed by written content. It would be interesting to validate this effect with a task with no propositional form, thus avoiding the problem of people with dyslexia having difficulties reading the premises, due to their specific literacy problem. This would be a more suitable approach to studying the inference process in reasoning.

Additionally, Bacon and Handley's (2010) results would indicate that participants with dyslexia may use the visual strategy without receiving any benefit from the content, which those without dyslexia can organise spatially. Furthermore, results indicated that people with dyslexia normally add physical characteristics to the premises, even when the terms given are relatively abstract. This addition could distract them from reaching an appropriate solution for the premises. Bacon, Handley, and McDonald (2007) claimed that ease of visualisation of the premises is the reason for people with dyslexia having problems, as the majority of their participants used a strategy that confused their reasoning.

Thus, these previous studies have shown singularities in the way people with dyslexia reason. However, with other reading disabilities (e.g., comprehension difficulties, nonspecific reading disabilities, like those in children with previous oral language problems or specific language impairment), there is less information about how these can influence reasoning strategies. Therefore, it is not known whether the differences in performing reasoning tasks found between people with and without reading disabilities are specific to components of text comprehension, limitations in working memory (phonological or visuospatial), or the process of reasoning.

This investigation, comparing elements of both reading and reasoning, should offer new knowledge on the scientific background related to reading and reasoning skills in a typical developing population. This study is a first step towards further investigation. To start with, it will focus on typically developed readers, thereby trying to clarify what is expected in a population without difficulties. Findings could lead to future research on reasoning in populations with reading difficulties.

The aim of the current study is to create a task similar to the traditional propositional transitive inference task, but reducing the reading (propositional) requirements. In a few studies, other deductive tasks were adapted to use diagrammatic and graphical premises in order to avoid the use of propositional premises. Moreno-Ríos and GarcíaMadruga (2002) used a task of this type with adults to test priming effects during deduction. Also, Moreno-Ríos, Rojas-Barahona, and García-Madruga (2014) used graphical premises to test differences in deduction between children, adolescents, and adults. These tasks showed similar general deductive effects, but allowed the propositional processing of the premises, which were irrelevant to the objective of the task, to be eliminated. Even though inference processing should be the same, the new task based on pictures should also show the visual impedance effect. The new task is designed to be very simple, and could be used in reasoning research for both adults and children with difficulties in reading and writing, without the interference of written language.

More specifically this study aims to:

- Design a very easy task of transitive reasoning with no propositional content in order to study reasoning skills and validate the visual impedance effect without the need for written language.

- Investigate the relationship between transitive reasoning and reading abilities and other related abilities like working memory and visual memory.

It is hypothesised that the new task, although using only pictorial and oral stimuli, will work similarly to the traditional propositional task for studying reasoning skills. Thus, the new task should work in finding the main reasoning effects, namely, validity (better performance in tasks with valid problems than those with invalid ones), and complexity (better performance in simple problems than in complex ones). Moreover, it is hypothesised that the new task, like the traditional propositional task, would be sensitive to the detection of the visual impedance effect. 


\section{METHOD}

\section{Participants}

Sixty-one adults (50 women, 11 men, age range: $18-44$ years), all students at undergraduate and postgraduate level, were contacted in their classes and provided information about the experiment. Participation was voluntary and offered extra marks in their courses as a reward for participation. All participants were native Spanish speakers, typically developed readers, without reading disabilities, as assessed through a previous interview and several reading tasks.

\section{Design}

A $2 \times 2 \times 2 \times 2$ (Imaginability $\times$ Validity $\times$ Complexity $\times$ Task) mixed design with four factors was carried out, using three within-subject factors (imaginability, validity, and complexity) and one between-subjects factor (task). The variable of imaginability had two levels: neutral and imaginable (problems); validity had two levels: valid and invalid (problems); complexity had two levels: simple and complex (problems); finally, task had two levels: propositional and picture (task).

\section{Materials}

Reasoning, reading, visual processing, and other basic cognitive skills (intelligence and memory) were tested as control measures. All tests were administered in the Spanish language.

\section{REASONING}

Participants completed two versions of a reasoning task: One written (propositional task) and the other visual/nonwritten (picture task). Eight different questionnaires were designed, randomising the order of the problems in the tasks. Half of the participants $(N=31)$ completed the propositional task first and the other half $(N=30)$ - the visual nonwritten task (picture task). However, only the first task was considered in the current study, because the second could have been influenced by previous experience. We were interested in the participants' impressions of using different strategies with the two tasks. No differences were found.

In each task version, participants had to solve 16 three-term series problems, displayed in random order. Eight problems included adjectives (translated from English to Spanish, aiming to replicate previous studies in English) that were easily imaginable, according to previous tests performed by Knauff and Johnson-Laird (2002; ugly-pretty; clean-dirty) and Bacon, Handley, and Newstead (2005; tall-short; rough-smooth). The other eight morphologically equal problems included neutral adjectives (smart-dumb from Knauff \& Johnson-Laird, 2002, and kind-cruel, rich-poor from Bacon et al., 2005). Half of the imaginable problems had a valid conclusion (i.e., when the premises are true, the conclusion must also be true); the other half had an invalid conclusion (i.e., the conclusion is not true, given that the premises are true, or there is no conclusion). Among the valid problems, two were simple problems (including the same adjective in both premises) and the other two were complex problems (including opposite adjectives in the two premises). A similar classification was developed for invalid problems and neutral problems.

Propositional task. Participants were presented with written premises that they had to read aloud. They then had to conclude what the relationship was between the last two terms in the context of the related adjectives given. They were asked to write the conclusion in the blank space under the written problem; this blank space could also serve to write down any other information (about the premises), helping to explain their reasoning process. The 16 problems were presented in a booklet, one per page, with space given under each for participants to write. An additional practice problem was used to explain the task.

Table 1 shows an example of a valid simple problem, an invalid simple problem, a valid complex problem, and an invalid complex problem in the propositional task.

-

TABLE 1.

Examples of Problems in the Propositional Task

\begin{tabular}{cc}
\hline Valid simple problem & Invalid Simple Problem \\
\hline The dog is taller than the cat. & The dog is taller than the cat. \\
$\begin{array}{c}\text { The cat is taller than the monkey. } \\
\text { The monkey is taller than the cat. } \\
\text { What can we say about }\end{array}$ & What can we say about \\
the dog and the monkey? & the dog and the monkey? \\
\hline Valid complex problem & Invalid complex problem \\
\hline The dog is taller than the cat. & The dog is taller than the cat. \\
$\begin{array}{c}\text { The monkey is shorter than the cat. } \\
\text { What can we say about }\end{array}$ & What can we say about \\
the dog and the monkey? & the dog and the monkey? \\
\hline
\end{tabular}

Picture task. Participants were presented with pictures instead of written premises and asked to solve the same 16 three-term series problems, also displayed in random order for each participant. Black pictures presented in cardboard squares with a white background $(3 \times$ $3 \mathrm{~cm}$ ) were used. Pictures of a dog, a cat, and a monkey were used (the same animals used in the propositional task). Moreover, pictures of a square (more) and a circle (less) were used (black cardboard squares, 1 $\times 1 \mathrm{~cm}$ ). This was aimed at aiding participants in symbolising the idea of more (or the opposite less) included in the premises. Although the premises only used the term more, less could be used by participants if they chose to. For this reason, two different elements (square and circle) were provided to represent the two ideas. The picture task did not use any written information. Premises were read aloud to the participants, who had to listen and use the pictures to "represent" them. Figure 1 shows an example of a valid problem constructed by a participant in the picture task.

\section{READING}

Participants completed two reading tests. The text comprehension subtest from the Bateria de Evaluación de los Procesos Lectores (PROLEC-SE Battery, Ramos \& Cuetos, 1999), measuring reading comprehension, was used. Participants had to read two texts followed by 10 questions on each text and write down their answers. Half of the questions were literal and the other half were inferential. The test scores ranged from 0 to 20 points. Reading time for each text was also measured (in seconds). 

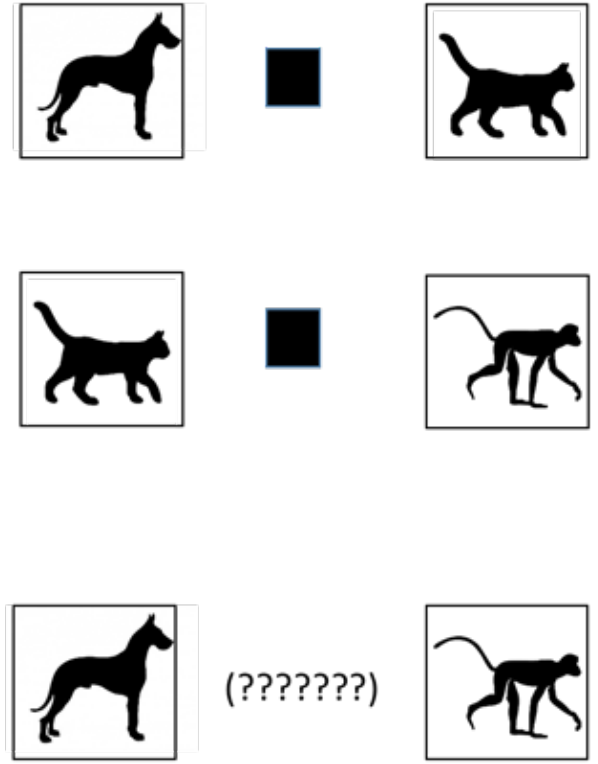

(???????)

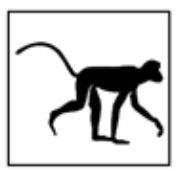

\section{FIGURE 1.}

Example of a valid simple problem with pictures in the Picture task. In this problem, participants listened to these premises: "The dog is taller than the cat. The cat is taller than the monkey. What can we say about the dog and the monkey?"The premises were provided only orally.

The Word Attack test from the Woodcock-Johnson III NU Tests of Achievement (McGrew \& Woodcock, 2001) evaluated the participant's phoneme/grapheme awareness, both in phonological and orthographical procedures. Participants read 28 pseudowords; including two practice items. The test scores ranged from 0 to 28 points. Time measures were also taken (in seconds).

\section{VISUAL PROCESSING}

Participants completed two visual processing tasks. The Corsi Cubes (McLean \& Hitch, 1999) measured visuospatial working memory, concentration, and attention. Participants were presented with nine cubes ( $2.5 \mathrm{~cm}$ each), randomly arranged on a board of $25.4 \times$ $27.94 \mathrm{~cm}$. Only the examiner could see the cubes, numbered 1 to 9 . The examiner presented a sequence (two to nine elements), increasing the complexity. Participants had to reproduce it. Each trial included two sequences. The task stopped when the participant failed to correctly complete both sequences of one trial. Both the direct and inverse versions of the task were used. Each version had a maximum score of 16 points; total score was the sum of both.

The Visual Patterns Test (VPT, Della Sala, Gray, Baddeley, Allamano, \& Wilson, 1999) evaluated visual working memory. Participants were presented with a chequerboard pattern for $3 \mathrm{~s}$ and had to reproduce it on a blank grid of the same size and shape as the pattern. The grids advanced in size, from the smallest, a $2 \times 2$ matrix (with two filled squares), to the largest, a $5 \times 6$ matrix (with 15 filled squares). There were three patterns at each complexity level. A pattern was correct when all the squares were appropriately represented in the grid. Testing stopped at the time when the participant failed to represent any of the three patterns at a given level of complexity correctly on the grid. Total score was calculated as the mean number of filled squares correctly recalled in the last three patterns recalled entirely correctly.

\section{INTELLIGENCE}

Raven's Progressive Matrices - General Scale (Raven, 2000) was used to measure the participants' nonverbal intelligence. Raw scores were measured. The test scores ranged from 0 to 60 points.

\section{MEMORY}

Digit Span from the Wechsler Intelligence Scale (WISC-R, Wechsler, 1974) — with both Digits Forward and Digits Backward subtests-was used. The maximum score was 28 points.

\section{Procedure}

All tests were individually applied in two sessions (approximately $1 \mathrm{~h}$ each). Each reasoning task was presented in a different session (counterbalanced order) and was always the first task performed in the session. Both reasoning tasks presented the same set of problems. The rest of the tests were distributed in the two sessions.

For the propositional task, students read the problems aloud, wrote down both their thoughts and the conclusion (written protocol) and detailed their reasoning process aloud while writing. After writing their conclusions, participants were also asked to explain aloud how they got to that conclusion. The session was recorded with a digital camera.

For the picture task, the examiner read the problems aloud while the participant looked at a display of 20 pictures placed in front of him/ her on the table (four images of a dog, four images of a cat, and four images of a monkey; four circles and four squares). Pictures were placed in three different columns: the dog pictures in the first column, the cat pictures in the second, and the monkey pictures in the third. Circles and squares were located in columns next to them. While listening to the premises, participants had to pick up the pictures from the columns and move them on the table in such a way that each premise was represented; the same procedure was required to represent their conclusion. Participants were also asked to describe their reasoning aloud while performing the task, and after finishing, explain how they got to that conclusion. The session was recorded with a digital camera.

For the remaining tests, normalised instructions were followed. Testing took part in a quiet laboratory.

\section{RESULTS}

A $2 \times 2 \times 2 \times 2$ (Imaginability $\times$ Validity $\times$ Complexity $\times$ Task $)$ mixed analysis of variance (ANOVA) with task as a between subjects factor, was carried out. Only accuracy data were analysed. Table 2 shows the results in both propositional and picture reasoning tasks. 


\section{TABLE 2.}

Mean Percentages of Correct Responses (M) and Standard Deviation (SD) as a Function of Problem Type in Both Propositional and Picture Tasks

\begin{tabular}{|c|c|c|c|c|c|c|c|c|c|}
\hline \multirow{5}{*}{$\begin{array}{l}\text { Propositional } \\
\text { task }\end{array}$} & & \multicolumn{4}{|c|}{ Neutral } & \multicolumn{4}{|c|}{ Imaginable } \\
\hline & & \multicolumn{2}{|c|}{ Valid } & \multicolumn{2}{|c|}{ Invalid } & \multicolumn{2}{|c|}{ Valid } & \multicolumn{2}{|c|}{ Invalid } \\
\hline & & $M$ & $S D$ & $M$ & $S D$ & $M$ & $S D$ & M & SD \\
\hline & Simple & 92 & 23 & 95 & 20 & 94 & 21 & 92 & 23 \\
\hline & Complex & 79 & 38 & 76 & 41 & 81 & 31 & 74 & 41 \\
\hline \multirow{2}{*}{ Picture task } & Simple & 98 & 6 & 98 & 9 & 97 & 13 & 95 & 15 \\
\hline & Complex & 88 & 25 & 83 & 30 & 90 & 28 & 67 & 44 \\
\hline
\end{tabular}

A significant main effect of complexity was found; participants showed more correct responses in simple problems than in complex problems $\left(95 \%\right.$ vs $\left.80 \% ; F[1,59]=28.070 ; \eta^{2}=.322 ; p<.01\right)$. No significant main effects of imaginability, $F(1,59)=2.626 ; \eta^{2}=.043$; $p>.05$, or validity, $F(1,59)=2.349 ; \eta^{2}=.038 ; p>.05$, were found. Finally, there was no significant main effect of task, $F(1,59)=1.188$; $\eta^{2}=.020 ; p>.05$.

A significant interaction between imaginability and validity was found; $F(1,59)=4.058 ; \eta^{2}=.064 ; p<.05$. This shows the visual impedance effect in the invalid problems $(88 \%$ vs $82 \% ; F[1,60]=4.678$; $\left.\eta^{2}=.060 ; p<.05\right)$, that is, more accurate answers in neutral versus imaginable problems. There were no significant differences in the valid problems, $F(1,60)=0.128 ; \eta^{2}=.002 ; p>.05$.

A significant interaction between validity and complexity was also observed; $F(1,59)=4.547 ; \eta^{2}=.072 ; p<.05$. Participants gave more correct responses in valid difficult problems than in invalid difficult problems $\left(84 \%\right.$ vs $75 \% ; F[1,60]=4.324 ; \eta^{2}=.042 ; p<.05$. In simple problems, no significant effects were found, $F(1,60)=0.002 ; \eta^{2}=.000$; $p>.05$.

The analysis for each task was carried out separately to test whether the predicted effects were present in the new task.

\section{Picture Task}

A $2 \times 2 \times 2$ (Imaginability $\times$ Validity $\times$ Complexity) analysis of variance (ANOVA) showed significant main effects of validity; participants gave more correct responses in valid problems $(94 \%$ vs $86 \% ; F[1,29]=$ $\left.6.735 ; \eta^{2}=.188 ; p<.05\right)$ than in Invalid problems.

Participants gave more correct responses in neutral problems than in imaginable ( $92 \%$ vs $86 \%$ ), even though this effect of imaginability was only marginally significant, $F(1,29)=3.832 ; \eta^{2}=.117 ; p=.06$.

A significant main effect of complexity was also found; the participants gave more correct responses in simple problems than in complex problems (97\% vs $\left.81 \% ; F[1,29]=13.956 ; \eta^{2}=0,325 ; p<.01\right)$.

A significant interaction between validity, complexity, and imaginability was found; $F(1,29)=5.191 ; \eta^{2}=.152 ; p<.05$. In valid problems, only the effect of complexity was marginally significant, $F(1,29)$ $=3.702 ; \eta^{2}=.113 ; p=.06$. However, in invalid problems, there was a significant main effect of complexity; participants gave more correct responses in simple problems than in complex problems (97\% vs. $75 \%$; $\left.F[1,29]=13.767 ; \eta^{2}=.322 ; p<.01\right)$. Additionally, in invalid problems only, a significant effect of imaginability was found; participants gave more correct responses in neutral problems than in imaginable prob- lems $\left(91 \%\right.$ vs $\left.81 \% ; F[1,29]=4.767 ; \eta^{2}=.141 ; p<.05\right)$. This last result would support the finding of the visual impedance effect.

\section{Propositional Task}

A $2 \times 2 \times 2$ (Imaginability $\times$ Validity $\times$ Complexity) analysis of variance (ANOVA) showed a significant main effect of complexity; participants showed more correct responses in simple problems than in complex problems ( $93 \%$ vs $77 \% ; F[1,30]=14.162 ; \eta^{2}=.321 ; p<.01$ ).

No significant main effects of imaginability, $F(1,30)=0.033 ; \eta^{2}=$ $.001 ; p>.05$, or validity, $F(1,30)=0.134 ; \eta^{2}=.004 ; p>.05$, were found. There were no significant interactions.

\section{Correlations Between Tasks}

In order to observe how reasoning measures (correct responses in valid and invalid problems, complex and simple problems, and neutral and imaginable problems) were connected with reading and related cognitive measures, a Pearson's correlation analysis for each reasoning task was carried out separately.

Results showed that the processes applied in the two reasoning tasks could be different.

\section{PICTURE TASK}

The reasoning results correlated with intelligence and reading comprehension. The hardest conditions, complex and invalid problems, showed correlations. Thus, there was a significant correlation between intelligence and complex problems, $r(30)=.490$, and also a significant correlation between intelligence and invalid problems, $r(30)=.475$, as well as imaginable problems, $r(30)=.511$. The same was shown with reading comprehension, which correlated with complex problems, $r(30)=.460$, invalid problems, $r(30)=.446$, imaginable problems, $r(30)=.399$, and neutral problems, $r(30)=.441$. Finally, a significant correlation was found between the standard scores of the Digit Span and intelligence, $r(30)=.418$.

\section{PROPOSITIONALTASK}

Table 4 shows the correlation matrix for the propositional task. There was a significant correlation between the scores in the inverse trials of the Corsi blocks and the total of the correct responses in reasoning problems, particularly with the imaginable problems, $r(30)=.389$, and the complex problems, $r(30)=.358$. 


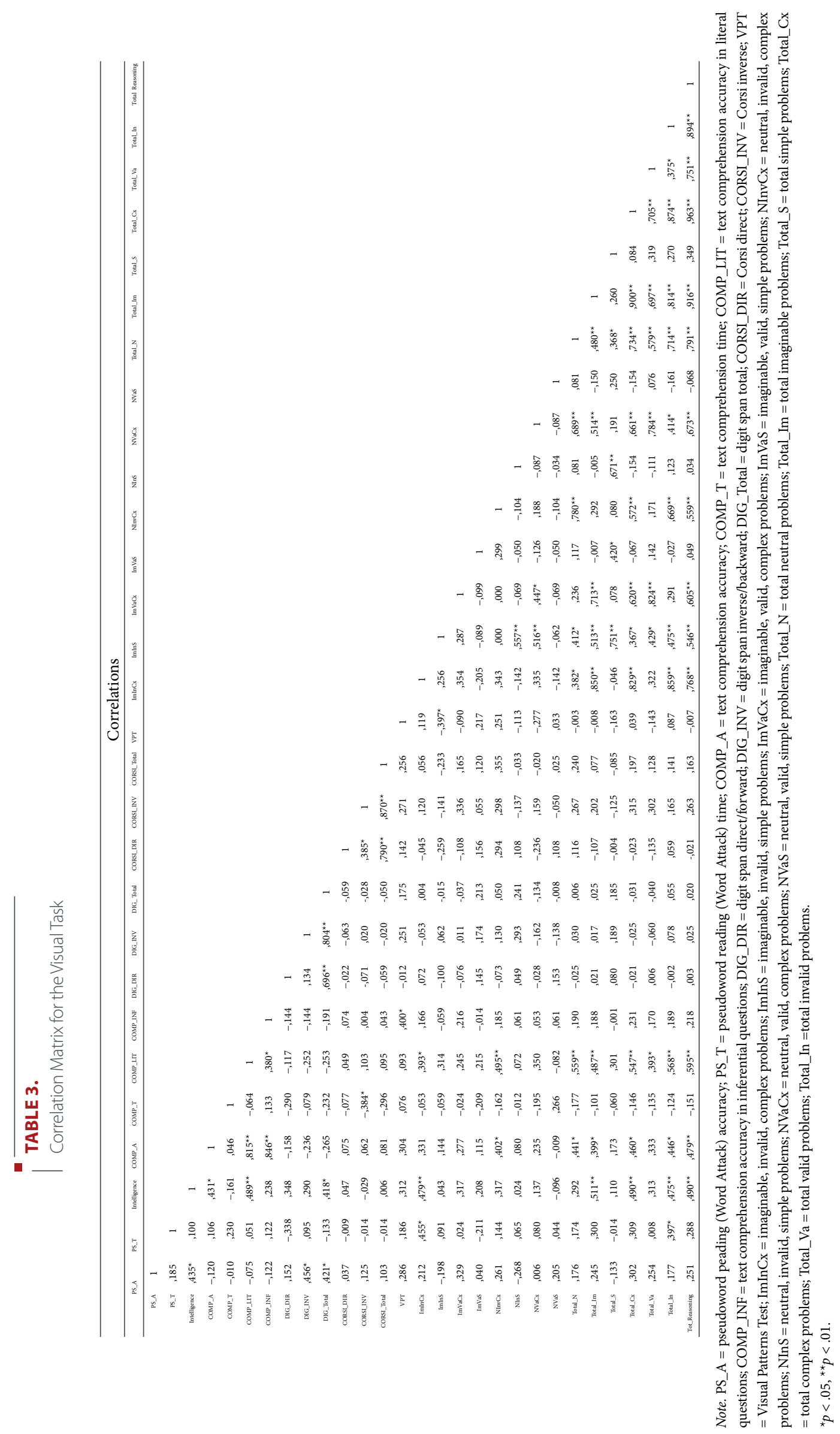



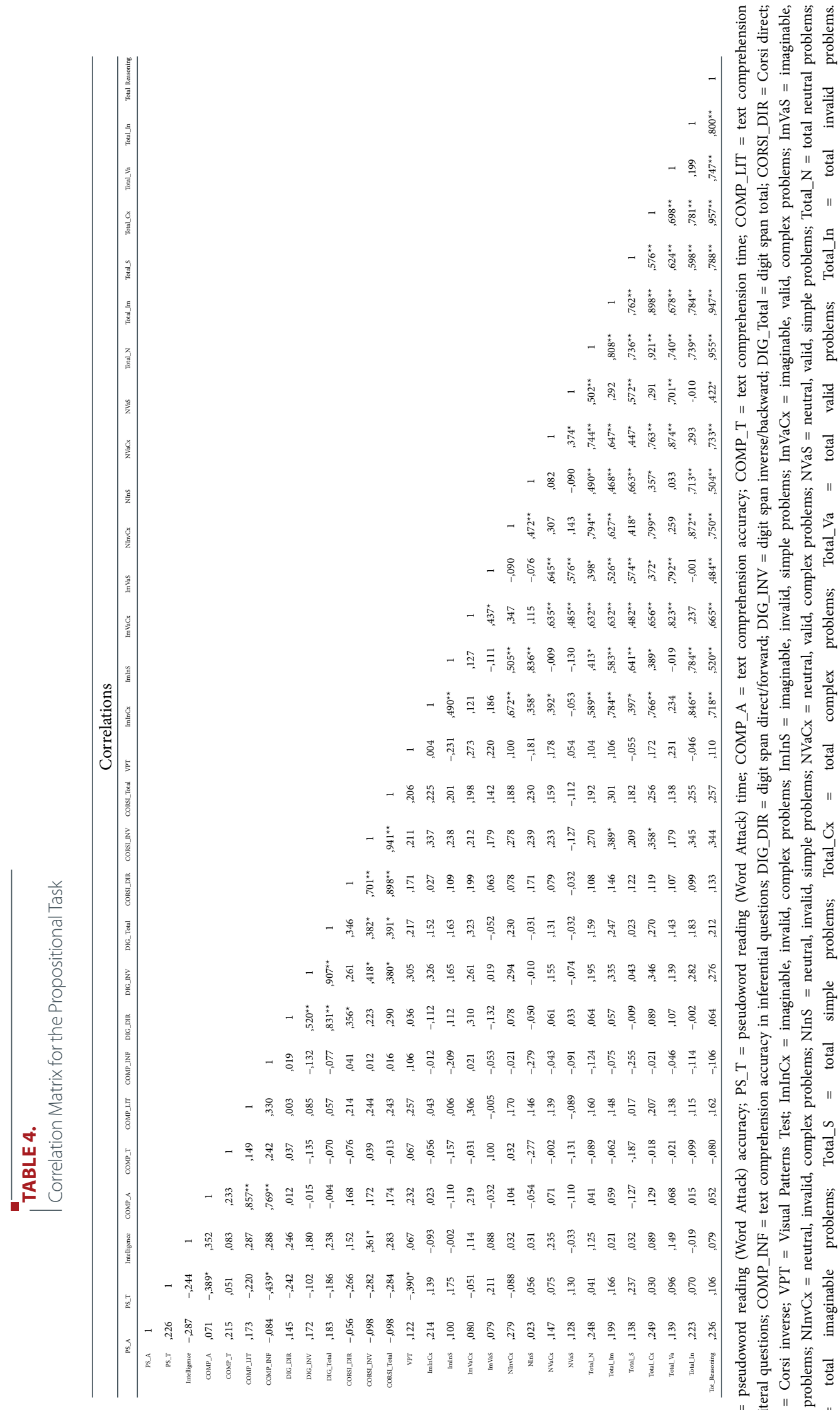

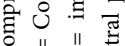

喜

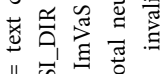

苯苍隐

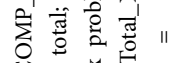

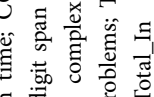
들

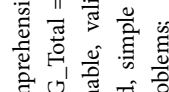

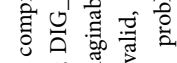

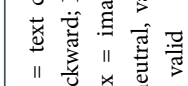
$H_{1} \stackrel{0}{0}$

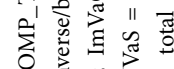
U.

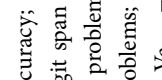

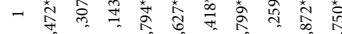

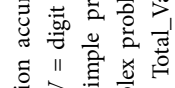
흘

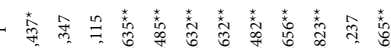

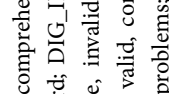

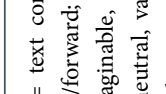

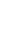
(1)

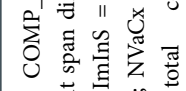

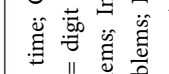

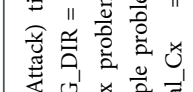

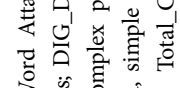

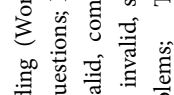

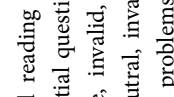

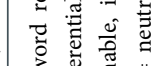

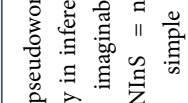
II

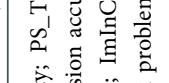

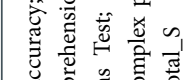

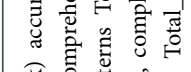

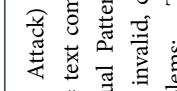

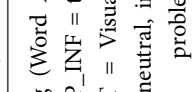

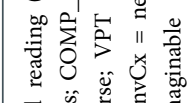

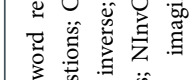

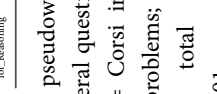

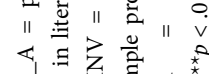

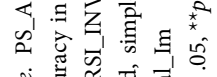

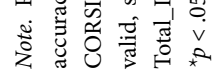




\section{DISCUSSION}

This study presents some new evidence for detection of the visual impedance effect (Knauff \& Johnson-Laird, 2002) by using an innovative reasoning task in which pictures are used instead of verbal content. Results have indicated that this new task is similar to the traditional propositional task used to measure transitive reasoning with simple problems. Results also indicated that the participants presented the visual impedance effect in the imaginable invalid difficult problems, showing that the picture task can be used to detect this effect. Actually, the traditional propositional task did not show this effect and only the complexity factor was significant. This could be due to the very simple problems used and the fact that participants were adults. The new task was more sensitive to detecting traditional effects, such as validity and the visual impedance effect. Even with this task, the visual impedance effect was shown only in the most difficult conditions, with complex and invalid problems.

Another aim of this study was to investigate the relationship between transitive reasoning, reading abilities, and other related abilities like working memory and visual memory. Diverse studies have revealed a connection between reasoning and reading comprehension in adults (Glenberg, Meyer, \& Lindem, 1987; Swanson, 2012), given that inference-making is essential to connect ideas and data that are not described in text (Cromley, et al., 2010). A variety of studies (Graesser, et al., 1994; Johnson-Laird, Legrenzi, Girotto, Legrenzi, \& Caverni, 1999; Kendeou, van den Broek, Helder, \& Karlsson, 2014) have shown that individuals spontaneously make inferences to compose a mental model from the conditions mentioned in the text while reading. The results of the present study are in line with previous results indicating an association between reasoning and reading comprehension in adult participants. Results with the new task showed that correlations between the most complicated reasoning conditions (complex problems, invalid problems, and, interestingly, the imaginable problems, which generate impedance) correlated with comprehension and with intelligence. More similar effects in the traditional propositional task would have been expected. However, it is possible that participants used a more automatic strategy to solve problems in this very simple, traditional task (System 1; see Khemlani \& Johnson-Laird, 2013). This could explain why there was no visual impedance effect and why the reasoning results do not correlate with comprehension and intelligence.

Additionally, some studies suggested a connection between reasoning and intelligence (i.e., Ackerman, Beier, \& Boyle, 2002), given that intelligence implicates compound cognitive processes like inductive and deductive reasoning (Colom, Flores-Mendoza, \& Rebollo, 2003); also, that reasoning capacity is a basic component in the formation of intelligence (Süß, Oberauer, Wittmann, Wilhelm, \& Schulze, 2002). The results of the present study agree with these previous findings, showing an association between reasoning and intelligence.

As in most studies of reasoning utilizing this task, differences by sex were not found (but see Wright \& Smailes, 2015, with children). In contrast, other spatial cognition tasks have demonstrated differences by sex, particularly in mental rotation (see Uttal et al., 2013), with better performance by males, while females performed better in verbal abilities (see Scheiber, Reynolds, Hajovsky, \& Kaufman, 2015). Some of these differences have been attributed to the use of different strategies of resolution (see Gold et al., 2018). Participants' reports in the present study did not allow for the detection of a differential use of strategies. However, caution should be applied to the interpretation of this absence of differences, because the number of women was much greater than that of men.

Finally, a variety of studies indicate a connection between working memory and intelligence (i.e., Ackerman, et al., 2002; Van Dyke, Johns, \& Kukona, 2014), suggesting that working memory is a factor of performance in cognitive tasks (Oberauer, Süß, Schulze, Wilhelm, \& Wittmann, 2000). The results of this study, obtained from the correlation analysis in the picture task, are in line with previous results, showing a connection between working memory storage capacity and intelligence.

Thus, this new picture task measuring reasoning has shown the effects of validity and complexity with very simple problems. In addition, it provides a measure of the visual impedance effect, which could help understand people's reasoning at different ages and with different reading abilities. It maintains the characteristics of other previously available reasoning tasks concerning related abilities, but it adds the value of a new measure free of literacy interference.

Consequently, it would seem to be a useful task for measuring reasoning, giving the opportunity to expand reasoning testing and offering possibilities beyond those of the previously available, traditional tasks.

Additional studies are needed to validate this task in other age groups, for example, in children. A task demanding lower literacy skills would also be suitable for studying reasoning skills at school levels before children have mastered writing skills. This also applies to special populations with written language problems (e.g., dyslexia, hearing problems and specific language impairment).

Moreover, the complexity (or simplicity) of reasoning problems should be considered. Although the aim was to design a very simple task, given that most of the problems were quite simple, participants could reach solutions easily, thus showing ceiling effects in some cases. Also, the information spoken out loud by the participants did not reveal enough data. Participants merely repeated the premises while reasoning. More complex problems would allow the experimenters to elicit some talk that could help in studying the reasoning process performed when participants think aloud.

\section{ACKNOWLEDGEMENTS}

The research reported in this work is partially funded by the Junta de Andalucía research group HUM 820 "LEE. Lectura y Escritura en Español,' FEDER Fundings, and the MINECO Project PSI201563505-P. 


\section{REFERENCES}

Ackerman, P. L, Beier, M. E., \& Boyle, M. O. (2002). Individual differences in working memory within a nomological network of cognitive and perceptual speed abilities. Journal of Experimental Psychology, 131, 567-589. doi: 10.1037/00963445.131.4.567 WWW

Bacon, A. M., Handley, S. J., \& Newstead, S. E. (2005). Verbal and spatial strategies in reasoning. In M. J. Roberts, \& E. J. Newton (Eds.), Methods of thought: Individual differences in reasoning strategies (80-105). New York City, NY: Psychology Press.

Bacon, A. M., Handley, S. J., \& McDonald, E. L. (2007). Reasoning and dyslexia: A spatial strategy may impede reasoning with visually rich information. British Journal of Psychology, 98, 79-92. doi: 10.1348/000712606X103987

Bacon, A. M., \& Handley, S. J. (2010). Dyslexia and reasoning: The importance of visual processes. British Journal of Psychology, 101, 433-452. doi: 10.1348/000712609X467314 WWW

Bacon, A. M., Parmentier, F. B. R., \& Barr, P. (2013). Visuospatial memory in dyslexia: Evidence for strategic deficits. Memory, 21, 189-209. doi: 10.1080/09658211.2012.718789 |WWW

Bacon, A. M., \& Handley, S. J. (2014). Reasoning and dyslexia: Is visual memory a compensatory resource? Dyslexia, 20, 330345. doi: 10.1002/dys.1483 WWW

Colom, R., Flores-Mendoza, C., \& Rebollo, I. (2003). Working memory and intelligence. Personality and Individual Differences, 34,

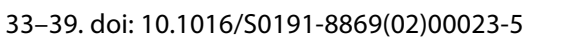

Cromley, J. G., Snyder-Hogan, L. E., \& Luciw-Dubas, U. A. (2010). Reading comprehension of scientific text: A domain-specific test of the direct and inferential mediation model of reading comprehension. Journal of Educational Psychology, 102, 687-700. doi: 10.1037/a0019452 WWW

Della Sala, S., Gray, C., Baddeley, A., Allamano, N., \& Wilson, L. (1999). Pattern span: A tool for unwelding visuo-spatial memory. Neuropsychologia, 37, 1189-1199. doi: 10.1016/S00283932(98)00159-6 WWW

Gazzo Castaneda, L. E., \& Knauff, M. (2013, January). Individual differences, imagery and the visual impedance effect. In M. Knauff (Chair). Proceedings of the Annual Meeting of the Cognitive Science Society. CogSci, vol. 35. Berlin, Germany.

Glenberg, A. M., Meyer, M., \& Lindem, K. (1987). Mental models contribute to foregrounding during text comprehension. Journal of Memory and Language, 26, 69-83. doi: 10.1016/0749596X(87)90063-5 WWW

Gold, A. U., Pendergast, P. M., Ormand, C. J., Budd, D. A., Stempien, J. A., Mueller, K. J., \& Kravitz, K. A. (2018). Spatial skills in undergraduate students-Influence of gender, motivation, academic training, and childhood play. Geosphere, 14, 668-683. doi:10.1130/ges01494.1 WWW

Graesser, A. C., Singer, M., \& Trabasso, T. (1994). Constructing inferences during narrative text comprehension. Psychological Review, 101, 371-395. |WWW

Johnson-Laird, P. N. (1983). Mental models. Cambridge, MA:
Harvard University Press.

Johnson-Laird, P. N. (2006). How we reason. Oxford, England: Oxford University Press.

Johnson-Laird, P. N, Legrenzi, P., Girotto, V., Sonino Legrenzi, M., \& Caverni, J-P. (1999). Naïve probability: A mental model theory of extensional reasoning. Psychological Review, 106, 62-88. doi: 10.1037/0033-295X.106.1.62 WWW

Johnson-Laird, P. N., \& Byrne, R. M. J. (2002). Conditionals: A theory of meaning, pragmatics, and inference. Psychological

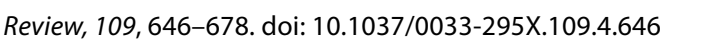
Kendeou, P., van den Broek, P., Helder, A., \& Karlsson, J. (2014). A cognitive view of reading comprehension: Implications for reading difficulties. Learning Disabilities Research \& Practice, 29, 10-16. doi: 10.1111//drp.12025 $\underline{\underline{W W} \mid}$

Khemlani, S., \& Johnson-Laird, P. N. (2013). The processes of inference. Argument and Computation, 4, 4-20. doi: 10.1080/19462166.2012.674060 $\underline{\underline{\mathrm{WWW}}}$

Knauff, M. (2018) Supporting and hindering effects on rational reasoning. In O. Zlatkin-Troitschanskaia, G. Wittum, \& A. Dengel (Eds.), Positive learning in the age of information (pp. 89-107). Wiesbaden, Germany: Springer VS.

Knauff, M., \& Johnson-Laird, P. N. (2002). Visual imagery can impede reasoning. Memory \& Cognition, 30, 363-371. doi: 10.3758/BF03194937 WWW

MacCullagh, L., Bosanquet, A., \& Badcock, N. A. (2017). University students with dyslexia: A qualitative exploratory study of learning practices, challenges and strategies. Dyslexia, 23, 3-23. doi: 10.1002/dys.1544 |WWW

McGrew, K. S., \& Woodcock, R. W. (2001). Technical manual. Woodcock-Johnson III. Rolling Meadows, IL: Riverside.

McLean, J. F., \& Hitch, G. J. (1999). Working memory impairments in children with specific arithmetic learning difficulties. Journal of Experimental Child Psychology, 74, 240-260. doi: 10.1006/ jecp.1999.2516 WWW

Moreno-Ríos, S., \& García-Madruga, J.A. (2002). Priming in deduction: A spatial arrangement task. Memory \& Cognition, 30, 1118-1127. doi:10.3758/BF03194329 WWW

Moreno-Ríos, S., Rojas-Barahona, C. A., \& García-Madruga, J. (2014). Perceptual inferences about indeterminate arrangements of figures. Acta Psyhcologica, 148, 216-225. doi: 10.1016/j.actpsy.2014.02.004 WWW

Oberauer, K., Süß, H.-M., Schulze, R., Wilhelm, O., \& Wittmann, W.W. (2000). Working memory capacity - facets of a cognitive ability construct. Personality and Individual Differences, 29,

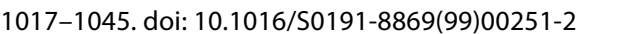

Osana, H. P., Lacroix, G. L., Tucker, B. J., Idan, E., \& Jabbour, G. W. (2007). The impact of print exposure quality and inference construction on syllogistic reasoning. Journal of Educational Psychology, 99, 888-902. doi: 10.1037/0022-0663.99.4.888 |www

Ramos, J. L., \& Cuetos, F. (1999). Batería de evaluación de los procesos lectores PROLEC-SE. [PROLEC-SE Battery. Assessment of reading processes]. Madrid, Spain: TEA Ediciones. 
Raven, J. (2000). The Raven's Progressive Matrices: Change and stability over culture and time. Cognitive Psychology, 41, 1-48. doi: 10.1006/cogp.1999.0735

Sato, Y., Sugimoto, Y., \& Ueda, K. (2017). Real objects can impede conditional reasoning but augmented objects do not. Cognitive Science, 42, 1-17. doi: 10.1111/cogs.12553 Ww

Scheiber, C., Reynolds, M. R., Hajovsky, D. B., \& Kaufman, A. S. (2015). Gender differences in achievement in a large, nationally representative sample of children and adolescents. Psychology in the Schools, 4, 335-348. doi: 10.1002/pits.21827 |WWW

Süß, H.M., Oberauer, K., Wittmann, W.W, Wilhelm, O., \& Schulze, R. (2002). Working-memory capacity explains reasoning abilityand a little bit more. Intelligence, 30, 261-288. doi: 10.1016/ S0160-2896(01)00100-3 WWW

Swanson, H. L. (2012). Adults with reading disabilities: Converting a meta-analysis to practice. Journal of Learning Disabilities, 45,

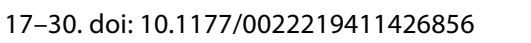

Uttal, D. H., Meadow, N. G., Tipton, E., Hand, L. L., Alden, A. R., Warren, C., \& Newcombe, N. S. (2013). The malleability of spatial skills: A meta-analysis of training studies. Psychological Bulletin, 139, 352-402. doi: 10.1037/a0028446 WWW

Van Dyke, J. A., Johns, C. L., \& Kukona, A. (2014). Low working memory capacity is only spuriously related to poor reading comprehension. Cognition, 131, 373-403. doi: https://doi. org/10.1016/j.cognition.2014.01.007 [wWw

Wechsler, D. (1974). Wechsler Intelligence Scale for ChildrenRevised. New York City, NY: Psychological Corporation.

Wright, B. C., \& Smailes, J. (2015). Factors and processes in children's transitive deductions. Journal of Cognitive Psychology, 27, 967-978. doi: 10.1080/20445911.2015.1063641 |wWW

RECEIVED 19.03.2018 | ACCEPTED 15.10.2018 